\title{
Análisis de calidad del agua para uso recreacional en la microcuenca del Arroyo Cuñapirú (Rivera, Uruguay)
}

La calidad de los cursos de agua depende de las variables ambientales, componentes de las cuencas y efectos antrópicos. Debido a que los sistemas fluviales son un importante recurso para la vida humana es imprescindible controlar la emisión de contaminantes, así como también realizar evaluaciones sistemáticas. La introducción de agentes extraños provenientes de la actividad humana puede modificar los ecosistemas alterando la calidad del agua. El presente trabajo comparó la calidad del agua de 15 estaciones de muestreo en el Arroyo Cuñapirú, cuantificando variables microbiológicas y parámetros fisicoquímicos. Las variables microbiológicas arrojaron valores elevados de coliformes fecales en la mayor parte de los muestreos, lo que indicó aportes elevados de nutrientes. Se apreció un correlación positiva entre la conductividad, oxígeno disuelto, nitratos y sulfatos (primer componente principal). Nuestros resultados evidencian la necesidad de una visión integral, multivariada, con el fin de comprender los efectos de las actividades humanas sobre los ecosistemas. Con este enfoque fue posible evaluar la calidad del agua con fines recreativos en la microcuenca del Arroyo Cuñapirú.

Palabras-chave: Sistema fluvial; Coliformes fecales; Parámetros fisicoquímicos.

\section{Water quality analysis for recreational use in the Arroyo Cuñapirú micro basin (Rivera, Uruguay)}

\begin{abstract}
The quality of water courses depends on environmental variables, watershed components and anthropic effects. Because river systems are an important resource for human life, it is essential to control the emission of pollutants, as well as to perform systematic evaluations. The introduction of foreign agents from human activity can modify ecosystems by altering water quality. The present work compared the water quality of 15 sampling stations in the Cuñapirú Stream, quantifying microbiological variables and physicochemical parameters. The microbiological variables showed high values of fecal coliforms in most of the samples, which indicated high nutrient intake. A positive correlation was observed between conductivity, dissolved oxygen, nitrates and sulfates (first major component). Our results show the need for a comprehensive, multivariate vision, in order to understand the effects of human activities on ecosystems. With this approach it was possible to evaluate the water quality for recreational purposes in the micro basin of the Cuñapirú Stream.
\end{abstract}

Keywords: River system; Fecal coliforms; Physic-chemical parameters.

Topic: Uso de Recursos Naturais

Reviewed anonymously in the process of blind peer.
Received: $12 / 10 / 2018$

Approved: $12 / 11 / 2018$
Juan Martín Rivero Simones

Universidad de la República, Uruguai

http://orcid.org/0000-0003-2436-6376

martinriverosimones@gmail.com

Andrés Canavero (iD

Universidad de la República, Uruguai

http://lattes.cnpq.br/1536057763340468

http://orcid.org/0000-0002-0044-6489

acanavero@gmail.com

Elena Castiñeira Latorre

Universidad de la República, Uruguai

http://lattes.cnpq.br/0996553120631176

http://orcid.org/0000-0002-8199-0215

elencasti@gmail.com
Referencing this:

SIMONES, J. M. R.; CANAVERO, A.; LATORRE, E. C.. Análisis de calidad del agua para uso recreacional en la microcuenca del Arroyo Cuñapirú (Rivera, Uruguay). Revista Ibero Americana de Ciências Ambientais, v.9, n.8, p.224-234, 2018. DOI: http://doi.org/10.6008/CBPC2179$\underline{6858.2018 .008 .0020}$

DOI: 10.6008/CBPC2179-6858.2018.008.0020 


\section{INTRODUCCION}

La calidad del agua está determinada por procesos naturales, acción de actividades humanas, así como las características de la cuenca. El escurrimiento superficial por parte de las precipitaciones, desechos vertidos por el hombre, la topografía, la vegetación y el tipo de suelo entre otros factores contribuyen a determinar el estado actual del ecosistema (AROCENA et al., 1996).

Debido a que los ríos, lagos y arroyos constituyen un importante recurso de agua dulce para consumo humano, industrial, agrícola y recreacional, es de suma importancia prevenir y controlar las fuentes de emisión de contaminantes, así como también monitorear las variaciones espaciales y temporales de la calidad del agua (TEIXEIRA, 2007). La introducción de agentes extraños al cauce, ya sean de origen orgánico o inorgánico afectará de forma negativa o positiva al sistema. Una evaluación sistemática de la calidad del agua es de vital importancia para mantener la salud ambiental.

Si bien la contaminación del agua ocasiona graves problemas a los seres vivos, en la mayoría de los casos se ha trabajado sistemáticamente con parámetros fisicoquímicos y microbiológicos, para su diagnóstico, análisis y evaluación. Las medidas físicas y químicas reflejan, el estado instantáneo del sistema, resultando necesario muestreos continuos de los cursos de agua en diferentes estaciones del año para poder caracterizar de forma general el sistema. Por otra parte, desde hace ya muchos años se ha empleado el recuento de coliformes como indicadores de contaminación en aguas y alimentos, por lo que sí están dentro de los parámetros estándares de control son aptas para su uso.

En Uruguay la contaminación de los ríos y arroyos es debido al aporte de materia orgánica y nutrientes inorgánicos (e.g. detergentes, fertilizantes). En algunas ocasiones esta puede ser de carácter puntual (e.g. origen urbano e industrial) o difuso (e.g. de origen agropecuario). Para evaluar las características físicas, químicas y biológicas del agua en el Uruguay se recurre a manuales elaborados por la Dirección Nacional de Medio Ambiente (DINAMA), Decretos como el 253/79 y guías de calidad de agua potable de la Organización Mundial de la Salud (OMS) donde se establecen normas y parámetros para prevenir la contaminación ambiental.

Aproximadamente en el año 1993, la Intendencia Departamental de Rivera comenzó a tomar muestras de aguas para análisis de calidad del agua del Arroyo Cuñapirú, el cual nace en la Cuchilla Negra, donde actualmente se encuentra la OSE del departamento y desemboca en el Río Tacuarembó. Al inicio se tomaban únicamente muestras del lago del Parque Gran Bretaña, del Club de Polo y un punto intermedio en Paso Bixico. Se consideraban que estos tres sitios cubrían una gran superficie territorial antes del ingreso del curso de agua a la ciudad. En principio se extraían muestras para realizar análisis de coliformes fecales (CF), posteriormente se buscó la existencia de Estreptococos sp. y CF.

Los puntos de muestreo fueron variando con el tiempo, debido al crecimiento de la población. Los criterios más relevantes empleados para comenzar a seleccionar las estaciones de muestreo fueron principalmente dos, cubrir todos los accesos de los cursos de agua a la ciudad y las condiciones de acceso 
para la toma de muestras por parte del personal inspectivo, de forma que se tornara más eficiente y efectivo el trabajo (Bertón, com. pers.).

En la actualidad ya se ha formado una red de muestreo, que abarca todos los cursos de agua que aportan al curso principal del Arroyo Cuñapirú y de esa forma se construye un diagrama de muestreo de la ciudad. Las 15 estaciones corresponden a: 'Laguna Piñeiro', 'Paso de la Estiva', 'Lagunón', 'Parque Gran Bretaña' (Playa), 'Parque Gran Bretaña' (Puerto), 'Club de Polo', 'Picada de Mora', 'Pedra Furada', 'Paso del Horno', 'Avda. Italia 2do Puente', 'Avda. Italia 1er Puente', 'La Raca', 'Barrio Bisio', 'Lagos del Norte' y ‘Paso Serpa'. El objetivo del presente trabajo fue determinar la calidad de agua a través de la cuantificación bacteriológica y fisicoquímica del Arroyo Cuñapirú, caracterizando en forma preliminar la calidad para uso recreacional, evaluando los coliformes fecales de acuerdo con estándares nacionales e internacionales.

\section{MATERIALES Y MÉTODOS}

La microcuenca del Arroyo Cuñapirú está contenida dentro de los límites departamentales, nace en la Cuchilla Negra (frontera con Brasil), al extremo noroeste del departamento de Rivera, hasta la frontera con Tacuarembó, donde confluye con el río Tacuarembó. El arroyo fluye de norte a sur y su cauce principal tiene un largo de $240 \mathrm{~km}$ aproximadamente, y su cuenca tiene una superficie de $3502 \mathrm{~km}^{2}$ (figura 1). Sus principales afluentes son los arroyos: Corrales, Mangrullo, Médanos, Curticeiras, Batoví y Ataques.

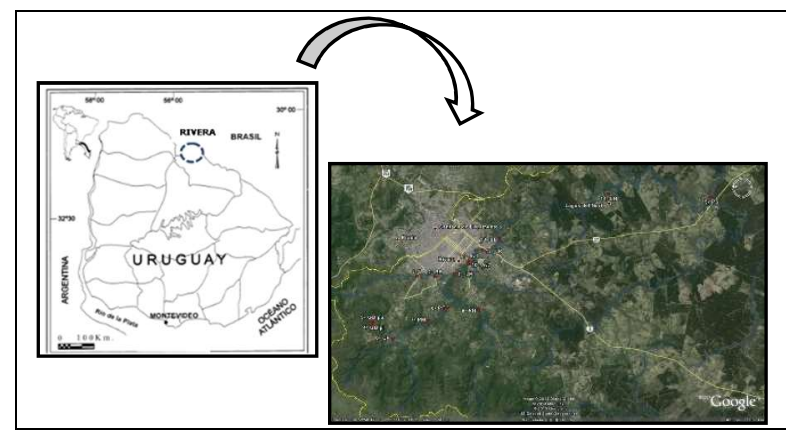

Figura 1: Ubicación del área de estudio y localización de los sitios de muestreo.

El departamento de Rivera esta subdividido en cinco cuencas hídricas, la del Río Tacuarembó, la del Arroyo Cuñapirú, la del Arroyo Yaguarí, la del Arroyo Caraguatá y la del Río Negro. Con respecto a las unidades de suelos de la cuenca del Cuñapirú estas son Rivera, cuchilla de Corrales, Tacuarembó y río Tacuarembó. Estas han sido destinadas a la forestación y cuentan con importantes emprendimientos de esta actividad; además la cuenca incluye la unidad Santa Clara, donde cuenta con actividades ganaderas. El departamento de Rivera está situado al norte del país, con una superficie de $9.379 \mathrm{~km}^{2}$.

Se localiza en latitud sur $30^{\prime} 54^{\circ}$ y su longitud oeste es de $55^{\prime} 31^{\circ}$. Limita al sur y suroeste con el departamento de Tacuarembó, al este con Cerro Largo, al oeste con Artigas y Salto y al norte y noreste con Brasil, (mayor frontera seca del país). El departamento cuenta con alturas considerables, de más de $350 \mathrm{~m}$ sobre el nivel del mar, sierras abruptas, cerros, bañados y valles con praderas (PRADERI et al., 2003).

Con respecto al contenido geológico, el departamento está conformado en la región NE por derrames basálticos correspondiente a la Formación Arapey, areniscas de la Formación Tacuarembó y Rivera en la 
región S y SE, la Isla Cristalina de Rivera en la región central del departamento conocida principalmente por la extracción de minería aurífera desde el siglo XIX, así como depósitos recientes/actuales. La superficie de Rivera representa un 5,35 \% del territorio nacional, cuenta con una población de 103.493 habitantes, dividiéndose en 95.891 en pobladores urbanos y 7.602 pobladores rurales. La actividad económica del departamento de Rivera es variada, siendo la producción principal la agricultura, seguida por la ganadería.

\section{Muestreo}

El muestreo se ejecutó de forma sistemática una vez al mes, realizándose en su totalidad 3 muestreos. En conjunto con la recolección de muestras, en las 15 estaciones se realizaron la toma de mediciones in situ de cuatro condiciones ambientales: temperatura, $\mathrm{pH}$, conductividad y oxígeno disuelto (OD) (tabla 1). Estos parámetros fueron medidos a través de cuatro equipos: 1-temperatura (ํㅜ, termómetro de laboratorio); 2-pH (Multiparámetro "Multilog Pro"); 3-Conductividad (Ms/cm, Multiparámetro "WTW ph/cond. 340i"); 4-OD (Mg/L, Sensor "Hanna HI 9146"). El tiempo de duración promedio en ejecutar toda la red de muestreo fue de seis horas en las tres instancias de campo.

Tabla 1: Se indican las 15 estaciones de muestreo, coordenadas geográficas e hidrodinámica del cuerpo de agua.

\begin{tabular}{|l|l|l|}
\hline Estación & Coordenadas Geográficas & Hidrodinámica \\
\hline $1-$-P & 30541265533317 & Lótico \\
\hline 2-PE & 30533645533457 & Lótico \\
\hline 4-LB.pl & 30530095533516 & Lótico \\
\hline $5-$ GB.pu & 30514745535525 & Léntico \\
\hline $6-$-P & 30514575535490 & Léntico \\
\hline $7-P M$ & 30523095536217 & Lótico \\
\hline $8-P F$ & 30533515535285 & Lótico \\
\hline $9-P H$ & 30541165534526 & Lótico \\
\hline $10-A V 2$ & 30551235534483 & Lótico \\
\hline $11-A V 1$ & 30543955533048 & Lótico \\
\hline $12-$-R & 30543825532587 & Lótico \\
\hline $13-B B$ & 30550245532296 & Lótico \\
\hline $14-$ LN & 30553285531574 & Lótico \\
\hline $15-P S$ & 30585545529405 & Léntico \\
\hline
\end{tabular}

Subtítulo: LP=Laguna Piñeiro, PE=Paso de la Estiva, L=Lagunón, GB.pl=Parque Gran Bretaña (Playa), GB.pu=Parque Gran Bretaña (Puerto), $C P=C l u b$ de Polo, $\mathrm{PM}=$ Picada de Mora, PF=Pedra Furada, $\mathrm{PH}=\mathrm{Paso}$ del Horno, AV2=Avda. Italia 2do Puente, AV1=Avda. Italia 1er Puente, LR=La Raca, BB=Barrio Bisio, LN=Lagos del Norte y PS=Paso Serpa.

\section{Fase laboratorio: Variables fisicoquímicas}

Las variables fisicoquímicas fueron identificadas a través del espectrofotómetro de absorción (Hach Lange DR2800), instrumento utilizado para la detección cuantitativa del azufre, cadmio, hierro, entre otros. Su campo de aplicación es diverso, ya sea análisis de agua, análisis de suelo, toxicología, farmacología, etc. Este método consiste en la medición de los distintos analitos por su absorción de onda particular; donde los electrones de los átomos en el atomizador son promovidos a orbitales más altos mediante la absorción de energía brindada por una longitud de onda conocida, obteniendo una señal proporcional a la concentración de elementos que se mide. Se tomaron como objeto de estudio el nitrato $\left(\mathrm{NO}_{3}{ }^{-}\right)$, nitrito $\left(\mathrm{NO}_{2}{ }^{-}\right)$, fosfato $\left(\mathrm{PO}_{4}{ }^{-}\right.$ ), sulfato $\left(\mathrm{SO}_{4}{ }^{2-}\right)$ y hierro ( $\left.\mathrm{Fe}\right)$; a través de los métodos presentados en el manual del equipo Hach Lange DR2800 (compuesto y método: $\mathrm{NO}_{3}{ }^{-}, 8039 ; \mathrm{NO}_{2}{ }^{-}, 8507 ; \mathrm{PO} 4,8048 ; \mathrm{SO}_{4}^{2}, 8051 ; \mathrm{Fe}, 8008$ ) (tabla 2). 
Tabla 2: Métodos utilizados en laboratorio para la identificación de parámetros fisicoquímicos según el manual Hach Lange DR2800. Unidades en $\mathrm{mg} / \mathrm{L}$.

\begin{tabular}{|l|l|}
\hline Parámetro & Método \\
\hline $\mathrm{NO}_{3}^{-}$ & 8039 \\
\hline $\mathrm{NO}_{2}^{-}$ & 8507 \\
\hline $\mathrm{PO}^{-}$ & 8048 \\
\hline $\mathrm{SO}_{4}^{2-}$ & 8051 \\
\hline $\mathrm{Fe}$ & 8008 \\
\hline
\end{tabular}

En el laboratorio se retiraba de cada muestra $10 \mathrm{~mL}$ de agua, a la cual se le adicionaba el reactivo, agitaba y dependiendo del método era el tiempo que necesitaba cada muestra para analizar. Este equipo reconoce cuantitativamente la presencia de los elementos en el agua a través de la 'comparación' de la muestra con reactivo y un blanco; el blanco es la muestra en su forma original, sin ningún agregado, en donde el aparato detecta la diferencia entre el blanco y la muestra con reactivo.

\section{Variables microbiológicas}

La concentración de bacterias indicadoras ha sido usada por décadas para evaluar la seguridad de las aguas recreacionales. Tales bacterias indicadoras no son necesariamente patógenas, pero predominan en desechos de origen humano donde es probable que existan organismos patógenos (FOLABELLA et al., 2006). Los criterios de calidad para agua de recreación están basados en la concentración de C.F.. Este grupo de bacterias son propias de la materia fecal y por lo tanto su presencia es un indicador cuantificable de contaminación; su origen se debe a aguas servidas sin tratamiento o tratamiento insuficiente, pluviales urbanas, establecimiento de crías de animales.

La determinación de CF fue a través de la técnica de 'filtración por membrana'. Esta técnica se utiliza para la determinación de CF en aguas naturales superficiales o subterráneas, aguas recreacionales y residuales, filtrando un volumen medido de la muestra, o la dilución adecuada de la misma y obteniendo el resultado en 24 horas. El principio de esta técnica consiste en la filtración de la muestra a través de una membrana de $\mathrm{NO}_{3}{ }^{-}$de celulosa y su incubación en una placa de Petri conteniendo M-FC Agar (medio de cultivo que permite el desarrollo de las bacterias coliformes).

Este medio de cultivo y la temperatura ideal de incubación de $44.5^{\circ} \mathrm{C}$, disminuyen el desarrollo de bacterias no coliformes que afectarían negativamente el crecimiento de los CF (DINAMA, 2009). Las Unidades Formadoras de Colonias (UFC) pasadas las 24 horas de incubación crecen con una coloración azul. El recuento de colonias se desarrolla en la propia placa en contraste con una lámpara incandescente para su mejor visibilidad; según el decreto 253/79, los cursos de agua destinados a uso recreacional no pueden superar las mil UFC, la media geométrica de cinco muestreos no debe exceder las 500UFC.

\section{Análisis estadísticos}

Con el fin de entender la variación conjunta de las variables fisicoquímicas de los sistemas acuáticos estudiados se realizó un Análisis de Componentes Principales (ACP). El ACP es una técnica estadística donde se sintetiza la información, es decir, ante un banco de datos con muchas variables, el objetivo será reducirlas a un menor número perdiendo la menor cantidad de información posible (QUINN et al., 2002). Luego 
realizamos análisis de regresión lineal entre el componente principal 1, que describe la mayor proporción de la variación del sistema fisicoquímico, como variable independiente y el logaritmo natural de la abundancia de coliformes como variable dependiente. Todos los análisis estadísticos se realizaron utilizando el software libre PAST Statistics Software (HAMMER et al., 2001).

\section{RESULTADOS}

Los valores registrados de $\mathrm{NO}_{3}^{-}$, en los tres muestreos en todas las estaciones, fueron inferiores a los estándares máximos permitidos por el Decreto de aguas del Uruguay (253/79), donde el valor máximo de $\mathrm{NO}_{3}{ }^{-}$en agua para uso recreacional en este es de $10 \mathrm{Mg} / \mathrm{L}$ (figura $2 \mathrm{~A}$ ). El valor más elevado de $\mathrm{NO}_{3}{ }^{-}$fue descubierto en el segundo muestreo en la estación 'Lagunón' (7,4Mg/L), correspondiente al mes de febrero; sin embargo, en todos los muestreos existieron casos donde no se registró la presencia de $\mathrm{NO}_{3}{ }^{-}$.

Si bien los $\mathrm{NO}_{2}^{-}$no son tomados en cuenta por el Decreto de aguas cuando hacemos referencia al uso recreacional, de todos modos si recurrimos a manuales de calidad de agua y los comparamos con los resultados obtenidos, el $\mathrm{NO}_{2}{ }^{-}$encontrado es bajo, ya que el mayor valor que se encuentra es de $0,117 \mathrm{Mg} / \mathrm{L}$ en 'Paso Serpa' correspondiente al mes de enero; y como en el caso anterior, existen muchas estaciones en todos los muestreos donde el $\mathrm{NO}_{2}{ }^{-}$es $\mathrm{OMg} / \mathrm{L}$ (figura $2 \mathrm{~B}$ ). Los valores de $\mathrm{PO}_{4}^{-}$registrados a lo largo de todo el período de muestreos nos reveló valores elevados de esta sustancia en todas las estaciones, si los comparamos con los estándares del Decreto 253/79; en todos los casos el $\mathrm{PO}_{4}{ }^{-}$estuvo por encima del rango permitido $(0,025 \mathrm{Mg} / \mathrm{L})$ (figura $2 \mathrm{C}$ ) e (tabla 3$)$.

$\mathrm{El} \mathrm{SO}{ }_{4}^{2-}$ es un sustancia que el Decreto no lo toma en cuenta cuando a usos recreacionales del agua se refiere, pero sí, se lo encuentra en la OMS para potabilidad de agua, como valor máximo permitido de 250 Mg/L (OMS, 2006), siendo en este caso no significativo, ya que además de ser valores bajos (el máximo $15 \mathrm{Mg} / \mathrm{L}$ encontrado en el "Barrio Bisio"), este cuerpo de agua tiene su destino para uso recreacional (Fig. 2D). El Fe presenta un valor máximo de $4,64 \mathrm{Mg} / \mathrm{L}$ en el muestreo de febrero en la estación de 'Laguna Piñeiro', siendo el mínimo valor registrado de $0,35 \mathrm{Mg} / \mathrm{L}$ en el primer muestreo del 'Paso de la Estiva' (figura $2 \mathrm{E})$.

Con relación al análisis microbiológico realizado, donde se pasó a contabilizar la cantidad de UCF en el Arroyo Cuñapirú, este parámetro muestra una variación extremada entre las distintas localidades de muestreo; por lo que se trabajó los datos en logaritmo. La normativa uruguaya de calidad de agua, toma como valor máximo permitido 1000 UFC, por lo que en todos los muestreos la variación de valores fue muy amplia y nos demostró que existieron casos que traspasaban el Decreto, mostrando valores por encima de un millón de colonias ('La Raca' segundo muestreo) y estaciones que estaban aptos para uso recreacional y relativamente bajos, como el caso de 'Lagos del Norte' y ‘Parque Gran Bretaña Playa' donde en el segundo muestreo existieron únicamente 10UFC en ambos casos (figura $2 \mathrm{~F}$ ).

El potencial de hidrógeno tomado in situ en todos los muestreos realizados, no presenta problemas severos de alcalinidad o basicidad, ya que el Decreto expresa que el máximo permitido es de $\mathrm{pH}=8,5$, y lo máximo encontrado en el Arroyo Cuñapirú fue de pH=7,55 en 'Lagos del Norte' en el mes de febrero; sin 
embargo se observaron casos de acidez en relación al Decreto $(\mathrm{pH}=6,5)$, 'Paso de la Estiva' en el primer y último muestreo realizado nos reveló valores por debajo de la normativa, así como también la "Laguna Piñeiro" en enero (figura $2 \mathrm{G}$ ).

La normativa plantea que el OD del agua no puede presentar valores menores a $5 \mathrm{Mg} / \mathrm{L}$ para su uso recreacional. Solo se observó un registro por debajo de la norma $(3,71 \mathrm{Mg} / \mathrm{L})$ en el sitio "Barrio Bisio" durante el muestreo de enero (figura $2 \mathrm{H}$ ). Los valores de conductividad registrados en el Arroyo Cuñapirú, fueron relativamente bajos en comparación con otros trabajos (ETCHEBARNE, 2010), donde el máximo valor encontrado fue de $508 \mathrm{Ms} / \mathrm{cm}$ y la media registrada de todo el muestreo de $151 \mathrm{Ms} / \mathrm{cm}$ (figura 2I). El decreto de aguas no toma en cuenta la conductividad como parámetro criterioso para su evaluación. La temperatura no está incluida en el decreto de agua, su valor máximo encontrado fue de $28,85^{\circ} \mathrm{C}$ en el sitio 'Parque Gran Bretaña (Puerto)' en el mes de enero y el mínimo de $11^{\circ} \mathrm{C}$ en la 'Laguna Piñeiro' en marzo (figura 2J). En este caso la media nos dio un valor de $18,7^{\circ} \mathrm{C}$ en todo el muestreo.

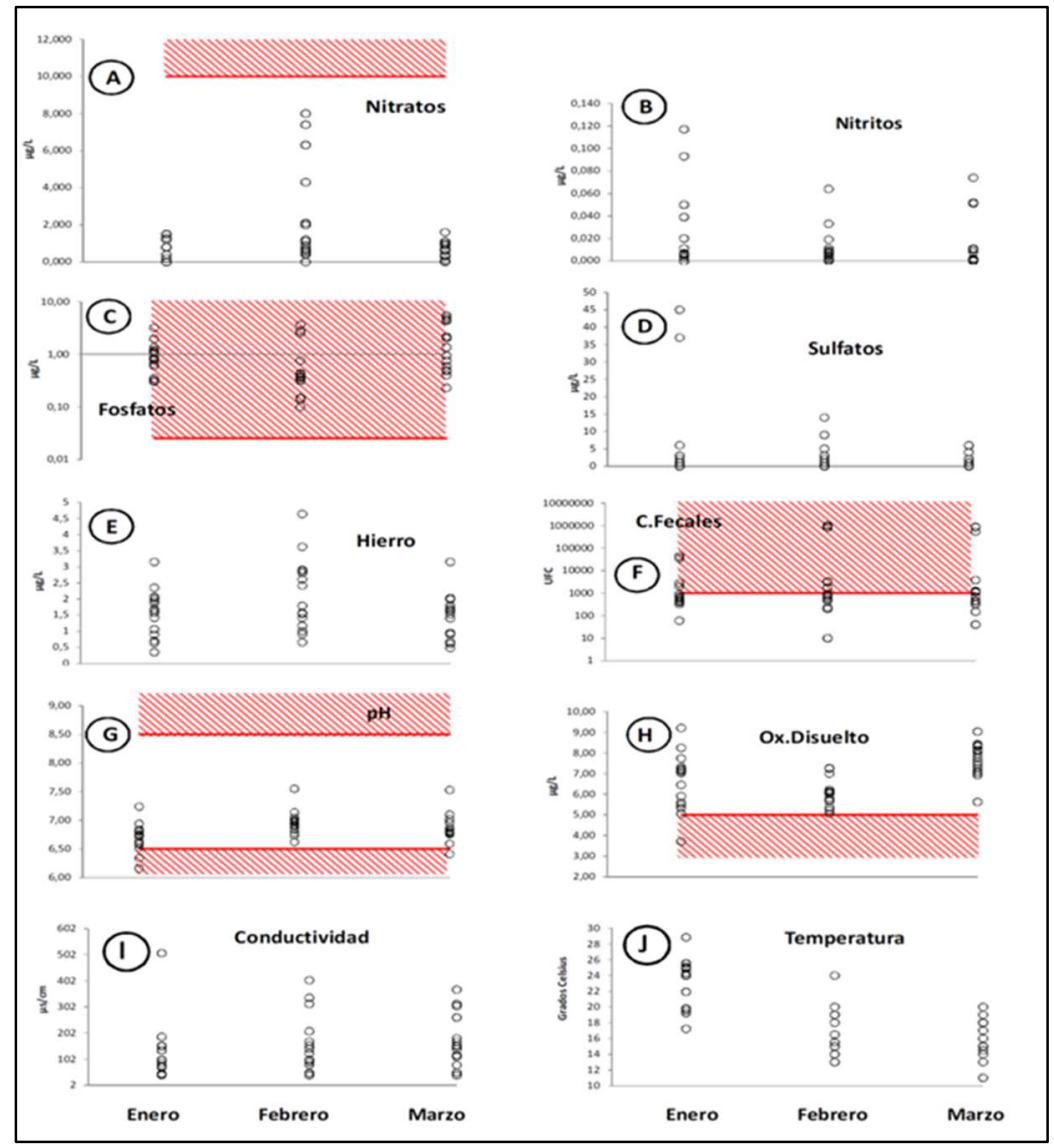

Figura 2: Variables fisicoquímicas registradas en los meses de enero, febrero y marzo del año 2013 para las 15 estaciones muestreadas. Las áreas sombreadas representan los valores no permitidos para el uso recreacional por el Decreto 253/79 para los parámetros: NO3-, PO4-, pH, OD y CF.

El Análisis de Componentes Principales (ACP), a partir de los dos primeros componentes representó el 63,2\% de la varianza total del sistema abiótico (figura 3). El Componente Principal 1 está representado en sus valores positivos por la conductividad, $\mathrm{PO}_{4}{ }^{-}, \mathrm{SO}_{4}{ }^{2-}, \mathrm{NO}_{2}{ }^{-}$asociados a los sitios Barrio Bisio (BB), Avenida Italia 1er Puente (AV1), y negativamente por el $\mathrm{NO}_{3}^{-}$y el OD asociado a Lagunón (L), Paso del Horno (PH) y Pedra Furada (PF) (figuras 3 y 4). Mientras que el Componente 2 fundamentalmente está representado por 
valores de correlación negativos de pH y temperatura asociados a los sitios Lagos del Norte (LN) y Paso Serpa (PS) y positivos en Laguna Piñeiro (LP) y Paso de la Estiva (PE) (figura 3).

Tabla 3: Cuantificación de variables fisicoquímicas y microbiológicas durante los tres ciclos de muestreo (mes 1-2-3).

\begin{tabular}{|c|c|c|c|c|c|c|c|c|c|c|c|c|}
\hline SITIOS & MES & InColif & Nitra & Nitrit & Fosfa & Sulfat & Hierro & Temp & $\mathrm{pH}$ & OxíD & Cond & ColiF \\
\hline LP & 1 & 6,08677473 & 0,200 & 0,000 & 1,20 & 37 & 3,15 & 24 & 6,16 & 8,24 & 135 & 440 \\
\hline $\mathrm{PE}$ & 1 & 7,92840603 & 0,000 & 0,000 & 1,11 & 0 & 0,35 & 25 & 6,34 & 7,17 & 187 & 2775 \\
\hline L & 1 & \begin{tabular}{|l|l|}
7,62070509 \\
\end{tabular} & 0,000 & 0,006 & 0,88 & 0 & 2,07 & 25,5 & 6,52 & 7,02 & 98 & 2040 \\
\hline GBpl & 1 & 6,27287701 & 1,500 & 0,011 & 0,30 & 6 & 1,64 & 25,57 & 6,60 & 5,91 & 45 & 530 \\
\hline Gbpu & 1 & \begin{tabular}{|l|}
4,09434456 \\
\end{tabular} & 1,500 & 0,006 & 1,18 & 3 & 1,57 & 28,85 & 6,58 & 6,45 & 44 & 60 \\
\hline $\mathrm{CP}$ & 1 & 6,01615716 & 1,300 & 0,003 & 0,77 & 1 & 0,64 & 24 & 6,64 & 7,31 & 71 & 410 \\
\hline PM & 1 & 5,99146455 & 1,500 & 0,007 & 1,96 & 1 & 0,9 & 19,84 & 6,72 & 5,59 & 75 & 400 \\
\hline PF & 1 & 6,55108034 & 0,000 & 0,005 & 0,60 & 0 & 1,06 & 19,6 & 6,76 & 7,10 & 86 & 700 \\
\hline $\mathrm{PH}$ & 1 & 6,04025471 & 0,800 & 0,039 & 0,35 & 2 & 1,88 & 17,23 & 6,72 & 7,22 & 98 & 420 \\
\hline AV2 & 1 & 6,39692966 & 0,800 & 0,003 & 0,32 & 3 & 2,35 & 21,92 & 6,74 & 5,32 & 135 & 600 \\
\hline AV1 & 1 & \begin{tabular}{|l|}
10,4385179 \\
\end{tabular} & 0,400 & 0,020 & 0,66 & 0 & 1,96 & 19,24 & 6,80 & 5,48 & 151 & 34150 \\
\hline LR & 1 & 10,6724377 & 0,800 & 0,050 & 1,35 & 1 & 2,04 & 24,33 & 6,84 & 7,73 & 152 & 43150 \\
\hline BB & 1 & 10,6689554 & 0,000 & 0,093 & 3,19 & 45 & 1,69 & 25,1 & 6,82 & 3,71 & 508 & 43000 \\
\hline $\mathrm{LN}$ & 1 & 6,79122146 & 0,000 & 0,003 & 1,04 & 1 & 1,42 & 24,9 & 7,24 & 9,21 & 40 & 890 \\
\hline $\mathrm{PS}$ & 1 & 5,768321 & 1,200 & 0,117 & 0,83 & 1 & 0,7 & 24 & 6,94 & 5,05 & 151 & 320 \\
\hline$\overline{\mathrm{LP}}$ & 2 & 6,13122649 & 0 & 0,019 & $0, \overline{0,31}$ & 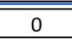 & 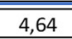 & 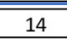 & $\bar{~} 6,78$ & $\bar{~} \overline{5,8}$ & 154 & 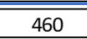 \\
\hline PE & 2 & 7,48436864 & 4,3 & 0,002 & 0,36 & 0 & 2,91 & 13 & 6,62 & 6,21 & 208 & 1780 \\
\hline $\mathrm{L}$ & 2 & 8,05515773 & 7,4 & 0 & 0,42 & 0 & 1,42 & 14 & 6,74 & 6,1 & 123 & 3150 \\
\hline GBpl & 2 & 2,30258509 & 2,1 & 0,009 & 0,14 & 3 & 1,57 & 19 & 6,98 & 6,08 & 47 & 10 \\
\hline Gbpu & 2 & 5,29831737 & 0,9 & 0,007 & 0,15 & 3 & 1,54 & 20 & 7 & 6,02 & 48 & 200 \\
\hline $\mathrm{CP}$ & 2 & 5,43807931 & 1,1 & 0,007 & 0,1 & 1 & 0,66 & 15 & 6,9 & 6,02 & 78 & 230 \\
\hline$P M$ & 2 & 6,82979374 & 0,6 & 0,004 & 0,43 & 1 & 1,17 & 15 & 6,92 & 7 & 89 & 925 \\
\hline PF & 2 & 6,69703425 & 8 & 0 & 0,44 & 1 & 1 & 15,5 & 6,92 & 7,24 & 97 & 810 \\
\hline $\mathrm{PH}$ & 2 & 6,50727771 & 6,3 & 0,001 & 0,33 & 14 & 2,88 & 13 & 6,92 & 7,26 & 99 & 670 \\
\hline \begin{tabular}{|l|} 
AV2 \\
\end{tabular} & 2 & 8,10167775 & 1,2 & 0,011 & 0,38 & 0 & 3,62 & 15,5 & 6,84 & 5,7 & 168 & 3300 \\
\hline \begin{tabular}{|l|} 
AV1 \\
\end{tabular} & 2 & 13,7431011 & 0,4 & 0,064 & 3,66 & 9 & 2,42 & 19 & 6,96 & 6,14 & 404 & 930150 \\
\hline LR & 2 & 13,8671538 & 0,5 & 0,008 & 2,81 & 2 & 2,81 & 18 & 7,04 & 5,22 & 313 & 1053000 \\
\hline BB & 2 & 13,6014506 & 0,7 & 0,011 & 2,56 & 5 & 2,61 & 16,5 & 7,04 & 5,67 & 337 & 807300 \\
\hline LN & 2 & 2,30258509 & 0,7 & 0,005 & 0,37 & 2 & 1,79 & 24 & 7,55 & 5,35 & 39 & 10 \\
\hline PS & 2 & 6,29156914 & 2 & 0,033 & 0,76 & 0 & 0,91 & 19 & 7,14 & 5,05 & 141 & 540 \\
\hline LP & 3 & $6,6,13122649$ & 0,7 & 0,002 & 5,01 & 0 & 1,99 & 11 & 6,86 & 7,03 & 167 & 460 \\
\hline PE & 3 & 5,768321 & 0,1 & 0 & 4,57 & 0 & 3,15 & 13 & 6,41 & 7,58 & 261 & 320 \\
\hline $\mathrm{L}$ & 3 & 7,05617528 & 0,3 & 0 & 0,94 & 0 & 1,4 & 15 & 6,59 & 8,37 & 150 & 1160 \\
\hline GBpl & 3 & 3,68887945 & 1 & 0,01 & 4,34 & 2 & 1,53 & 18 & 6,82 & 7,12 & 49 & 40 \\
\hline Gbpu & 3 & 3,68887945 & 1,6 & 0 & 0,48 & 0 & 1,63 & 19 & 6,86 & 6,92 & 49 & 40 \\
\hline $\mathrm{CP}$ & 3 & 5,79909265 & 0,4 & 0,001 & 0,59 & 2 & 0,67 & 15 & 6,78 & 7,27 & 78 & 330 \\
\hline PM & 3 & \begin{tabular}{|c|}
6,55108034 \\
\end{tabular} & 0,9 & 0,074 & 1,36 & 0 & 0,95 & 14,5 & 6,76 & 8,31 & 117 & 700 \\
\hline PF & 3 & 7,1428274 & 0,7 & 0,051 & 5,49 & 0 & 0,91 & 15 & 6,76 & 8,14 & 140 & 1265 \\
\hline $\mathrm{PH}$ & 3 & \begin{tabular}{|l|}
6,98933527 \\
\end{tabular} & 0,6 & 0,002 & 0,76 & 1 & 0,61 & 14 & 6,8 & 9,03 & 111 & 1085 \\
\hline AV2 & 3 & \begin{tabular}{|l|}
8,24275635 \\
\end{tabular} & 1,1 & 0,001 & 0,23 & 0 & 1,7 & 16 & 6,76 & 8,42 & 181 & 3800 \\
\hline AV1 & 3 & \begin{tabular}{|c|}
13,6509307 \\
\end{tabular} & 0 & 0,052 & 2,03 & 6 & 1,63 & 18 & 6,78 & 8,08 & 368 & 848250 \\
\hline LR & 3 & 13,6848322 & 0,9 & 0,008 & 2,03 & 6 & 1,79 & 18 & 6,96 & 7,94 & 312 & 877500 \\
\hline BB & 3 & \begin{tabular}{|l|}
13,2213033 \\
\end{tabular} & 0 & 0,011 & 2,18 & 1 & 2,03 & 17 & 7,02 & 7,75 & 305 & 552000 \\
\hline LN & 3 & 6,06378521 & 1 & 0,001 & 0,4 & 4 & 1,64 & 20 & 7,53 & 5,62 & 39 & 430 \\
\hline PS & 3 & 5,01063529 & 1,1 & 0,011 & 0,49 & 1 & 0,48 & 18 & 7,1 & 7,45 & 154 & 150 \\
\hline
\end{tabular}

El análisis de correlación entre el Componente Principal 1 (variable independiente) extraído del ACP

(Fig. 3 y 4) y el logaritmo natural de la abundancia de coliformes (variable dependiente) reportó una asociación significativa entre estas dos variables $(p=0,005)$ describiendo el $46,9 \%$ de la varianza de la abundancia de los coliformes a lo largo del sistema fluvial (figura 5).

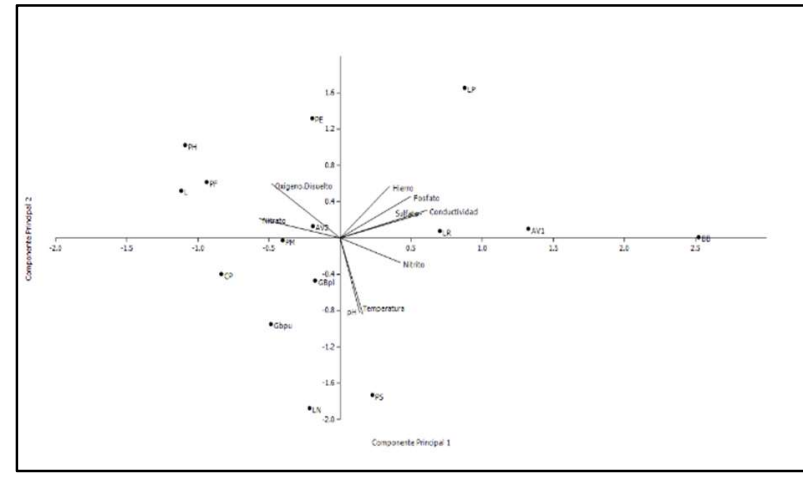

Figura 3: Representación de los dos primeros componentes del ACP. Componente principal 1 (36,6\% de varianza explicada) vs. Componente principal 2

(26,6\% de varianza explicada)

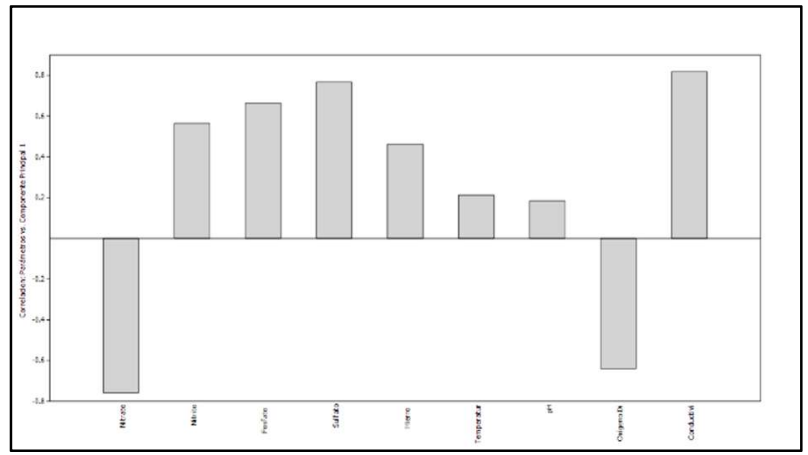

Figura 4: Correlación de las variables fisicoquímicas y condiciones ambientales en el Componente Principal 1. 


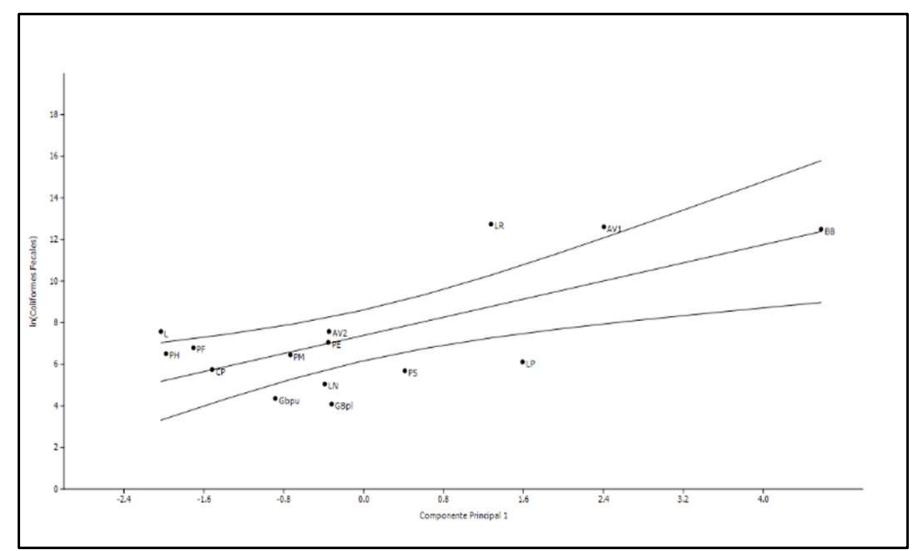

Figura 5: Correlación entre el Componente Principal 1 y el logaritmo natural de la abundancia de $C F(y=1,089 * x+7,393$; $R 2=0,469 ; p=0,005)$.

\section{CONCLUSIÓN}

En términos generales, los valores registrados de los parámetros que refieren a la calidad del agua se encuentran dentro de las normas legales (Decreto 253/79). Sin embargo, los valores de $\mathrm{NO}_{3}{ }^{-}$obtenidos son relativamente altos si los comparamos con otros trabajos (Perdomo et al., 2001). Ninguna estación de muestreo indicó estar por encima del rango permitido, reportándose un valor máximo en la estación "Lagunón" (7,4Mg/L) correspondiente al segundo muestreo.

El valor elevado de $\mathrm{NO}_{3}{ }^{-}$puede corresponder a fuentes de contaminación localizadas de restos orgánicos y de origen humano como las aguas servidas y cámaras sépticas (PERDOMO et al., 2001). La asociación observada entre el $\mathrm{NO}_{3}{ }^{-}$y lo $\mathrm{CF}$ fue inversamente proporcional. El $\mathrm{NO}_{2}{ }^{-}$indicó una muy baja variación de sus resultados a lo largo de todo el muestreo. En otros trabajos, las concentraciones de $\mathrm{NO}_{2}{ }^{-}$ fueron dependientes del pH (Camargo \& Alonso, 2007). Si bien no reportamos dicha relación en nuestra área de estudio, si se observó fuerte asociación entre este ión y el OD.

$\mathrm{El} \mathrm{PO}_{4}{ }^{-}$fue la principal variable encontrada por encima del rango permitido, donde todos los sitios de muestreo en todo el período se encontraron por encima del máximo tolerable para uso recreacional (10 $\mathrm{Mg} / \mathrm{L})$. El aumento de este ión en el medio acuático está relacionado a diversas actividades humanas, principalmente el uso de fertilizantes y detergentes. Esta sustancia constituye en muchos sistemas el nutriente limitante de la producción primaria, resultando determinante del estado trófico de los mismos (Arocena \& Conde, 1999). Los valores de $\mathrm{SO}_{4}{ }^{2-}$ fueron bajos para todo el período de muestreo, donde la OMS, para agua potable, establece como máximo $250 \mathrm{Mg} / \mathrm{L}$ (OMS, 2006); en ningún momento el máximo se aproximó a este valor.

El Fe registrado en el Arroyo Cuñapirú en el período de muestreo no superó en ningún momento los $5 \mathrm{Mg} / \mathrm{L}$, valor considerado no muy alto por la OMS, ya que el decreto uruguayo de aguas no lo toma en cuenta. Sí debe ser tomado en cuenta si el destino del Arroyo tuviera su uso destinado a abastecer de forma directa la población, ya que altas concentraciones de Fe en el organismo se pueden acumular dañando la salud. Otro factor importante en cuanto a Fe se refiere, que este ha comenzado a cobrar importancia en los sistemas de eutrofización de los cursos de agua (CAMARGO et al., 2007). 
La calidad microbiológica del agua presentó fuerte variación en todo el muestreo, parte de esta variación se explica por las concentraciones de $\mathrm{NO}_{3}{ }_{3}$, el cual es inversamente proporcional a las concentraciones de CF. Sin embargo, el pH y la temperatura fueron dos parámetros poco influyentes en dicha variación. Los cambios antrópicos pueden explicar parte de esta variación, debido a la materia orgánica vertida en desagües de pozos negros provenientes de la red de saneamiento, que junto con las precipitaciones hace elevar el nivel del curso e ingresar los restos materiales al sistema fluvial (Hong et al., 2010). Según el decreto nacional uruguayo, los cursos de agua destinados a la recreación no pueden exceder las 1000 colonias de CF, que sin embargo en este trabajo esos valores fueron superados en la mayor parte de los muestreos.

El pH reportó valores aceptables para la normativa, tendiendo a una mayor acidificación de los sistemas. Únicamente 3 muestras observaron valores de $\mathrm{pH}$ por debajo del valor indicado por el decreto $(6,5)$ y en ningún caso se encontró por encima del valor admitido $(8,5)$. Esta variable no influyó de forma significativa en la variación de los CF.

EI OD, que debe estar por encima de los $5 \mathrm{Mg} / \mathrm{L}$ en usos recreacionales, demostró encontrarse en casi su totalidad dentro de las normas permitidas, una única muestra presentó valores por debajo del rango permitido. El OD en el agua, que permite el desarrollo de vida acuática, demostró valores correctos y con buen potencial para la salud ambiental del sistema acuático.

La conductividad no reportó valores elevados en el sistema, comparándolos con los de Etchebarne (2010), observándose en un único caso $508 \mathrm{Ms} / \mathrm{cm}$, siendo los restantes por debajo de este valor. En este caso, cuando los valores de conductividad aumentan, los CF también crecen, demostrando como los ecosistemas acuáticos pierden calidad cuando el OD es bajo y la conductividad es alta, lo que determina condiciones desfavorables para la biota (OCON et al., 2012).

La temperatura tuvo variaciones a lo largo del muestreo, lo que puede haber influenciado en las variables fisicoquímicas por la solubilidad de estas, según el $A C P$, esta no demuestra ser incisiva en el aumento o disminución de CF. La temperatura del agua tiene una gran importancia en el desarrollo de los diversos procesos que en ella se realizan, de forma que un aumento de la temperatura modifica la solubilidad de las sustancias, aumentando la de los sólidos disueltos y disminuyendo la de los gases (Jiménez, 2000).

Si bien la abundancia de CF está determinada por la cantidad de materia orgánica y desechos urbanos, en este trabajo las principales variables asociadas al aumento de los CF fueron la conductividad, $\mathrm{OD}, \mathrm{NO}_{3}{ }^{-} \mathrm{SO}_{4}{ }^{2-}$. Hong et al. (2010) resaltan la importancia de incorporar en el análisis la variación ambiental estacional, principalmente la precipitación, la cual en este trabajo no ha sido incluida. Si el sistema de aguas tiene un destino recreacional, las variables que más repercuten en la inestabilidad del Arroyo son el $\mathrm{PO}_{4}^{-} \mathrm{y}$ los $\mathrm{CF}$, ya que estos son los 2 parámetros que más se alejan de lo que las normas legales permiten, teniendo las otras variables variaciones no muy fuertes en todos los muestreos.

Los análisis presentados en este trabajo contribuyen a evidenciar la necesidad de una visión integral del sistema, teniendo en cuenta las actividades humanas que modifican el sistema, así como también las variables naturales que juntas generan un cambio en el Arroyo Cuñapirú. Se torna necesario realizar nuevas 
investigaciones en el área incluyendo medidas del aporte de sedimentos y efluentes provenientes de modificaciones de origen humano, así como nuevas variables ambientales (e.g. precipitaciones) con registros estacionales. De esta manera se generará conocimiento que permita una mejora en el manejo de la cuenca, pudiéndose así conservar el recurso para su uso recreacional.

\section{REFERENCIAS}

AMPUDIA, L.; HERNÁNDEZ, M.. Desarrollo: enfoques y desafíos, Nóesis. Revista de Ciencias Sociales y

Humanidades, Juárez, v.19, p.51, 2010.

AROCENA, R.; CONDE, D.. Métodos en ecología de aguas continentales. Montevideo: Facultad de Ciencias, 1999.

AROCENA, R.; CONDE, D.; FABIÁN, D.; GORGA, J.; CLEMENTE, J.. Calidad de agua en la Laguna de Rocha: rol de sus principales afluentes. PROBIDES v.1, n.50, 1996.

CAMARGO, J. A.; ALONSO, A.. Contaminación por nitrógeno inorgánico en los ecosistemas acuáticos: problemas medioambientales, criterios de calidad del agua, e implicaciones del cambio climático. Ecosistemas, v.16, p.98110, 2007.

CHALAR, G.. Calidad ecológica de los cursos de agua. Montevideo: Universidad de la República, 1993.

DINAMA. Dirección Nacional de Medio Ambiente. Manual de Procedimientos Analíticos para Muestras Ambientales. Montevideo: DINAMA, 2009.

ETCHEBARNE, V.. Calidad de agua y de la zona ribereña en sistemas lóticos de regiones ganaderas extensivas y lecheras de la cuenca del Río Santa Lucía. Montevideo: Universidad de la República, 2010.

FOLABELLA, A.; ESCALANTE, A.; DEZA, A.; PEREZ, J.; ZAMORA, A.. Indicadores bacterianos de la calidad de el agua recreacional en la Laguna de los Padres. Buenos Aires: 2006.

HAMMER, $\varnothing$.; HARPER, D. A. T.; RYAN, P. D.. PAST:

Paleontological Statistics Software Package for Education \& Data Analysis. Palaeontologia Electronica v.4, n.9, 2001.
HONG, H.; QIU, J.; YAN, L.. Environmental factors influencing the distribution of total \& fecal coliform bacteria in six water storage reservoirs in the Pearl River Delta Region, China. Journal of Environmental Science, v.22, p.663-668, 2010.

JIMÉNEZ, A. A.. Determinación de los parámetros físicoquímicos de calidad de las aguas. Madrid: Universidad de Carlos, 2000

OCON, S.; RODRÍGUEZ, A.. Assessment of water quality in temperate-plain streams (Argentina, South America) using a multiple approach. Ecología Austral, v.1, p.81-91, 2012.

OMS. Organización Mundial de la Salud. Guías para la calidad del agua potable. Genebra: OMS, 2006.

PERDOMO, C. H.; CASANOVA, O. N.; CIGANDA, V. S.. Contaminación de aguas subterráneas con nitratos y coliformes en el litoral sudoeste del Uruguay. Agrociencia, v.5, p.10-22, 2001.

PRADERI, R. C.; VIVO, J. E.; PRADERI, F. V.. Ríos, lagos y montes indígenas del Uruguay. Montevideo: 2003.

QUINN, G. P.; KEOUGH, M. J.. Principal Components \& Correspondence Analysis, Experimental Design \& Data Analysis for Biologists. Cambridge: Cambridge University Press, 2002.

TEIXEIRA, F.. Efecto del uso del suelo sobre la calidad del agua y las comunidades de peces en sistemas lóticos de la cuenca baja del río Santa Lucía (Uruguay). Montevideo: Universidad de la República, 2007.

A CBPC - Companhia Brasileira de Produção Científica (CNPJ: 11.221.422/0001-03) detém os direitos materiais desta publicação. Os direitos referem-se à publicação do trabalho em qualquer parte do mundo, incluindo os direitos às renovações, expansões e disseminações da contribuição, bem como outros direitos subsidiários. Todos os trabalhos publicados eletronicamente poderão posteriormente ser publicados em coletâneas impressas sob coordenação da Sustenere Publishing, da Companhia Brasileira de Produção Científica e seus parceiros autorizados. Os (as) autores (as) preservam os direitos autorais, mas não têm permissão para a publicação da contribuição em outro meio, impresso ou digital, em português ou em tradução. 